\title{
Small molecule-enrichment analysis in response to osmotic stimuli in the intervertebral disc
}

\author{
H. Ma ${ }^{1 *}$, Y.Z. Xie ${ }^{2 *}$, J. Zhao ${ }^{2}$ and B. $\mathrm{Ye}^{1}$ \\ ${ }^{1}$ Department of Orthopaedic Surgery, Shanghai First Rehabilitation Hospital, \\ Shanghai, P.R. China \\ ${ }^{2}$ Department of Orthopaedic Surgery, Ninth People's Hospital, \\ Shanghai Jiaotong University School of Medicine, Shanghai, China \\ *These authors contributed equally to this study. \\ Corresponding author: B. Ye / J. Zhao \\ E-mail: painless_mh@126.com
}

Genet. Mol. Res. 11 (4): 3668-3675 (2012)

Received May 9, 2012

Accepted October 1, 2012

Published October 9, 2012

DOI http://dx.doi.org/10.4238/2012.October.9.2

\begin{abstract}
The intervertebral disc (IVD) is a heterogeneous structure that contributes to load support and flexibility in the spine. IVD cells experience a broad range of physical stimuli under physiological conditions, including alterations in their osmotic environment. To date, the molecular mechanisms regulating the response of IVD to osmotic pressure are still not well understood. We obtained the gene expression profile of human IVD cells from NCBI and looked for potential therapeutic drug candidates. Based on microarray data, we concluded that RAP2A and GNA13 appear to have a role in response to osmotic stimuli in intervertebral discs. Using a computational bioinformatics method, we determined that thioridazine has potential as a therapeutic drug candidate for regulating osmotic pressure changes in IVD cells. We anticipate that our results will be used to generate hypotheses for laboratory, patient, and population-based studies.
\end{abstract}

Key words: Intervertebral disc; Osmotic pressure; Small molecules 


\section{INTRODUCTION}

The intervertebral disc (IVD) is an avascular and alymphatic tissue that consists of three morphologically distinct zones, namely the annulus fibrosus, nucleus pulposus, and transition zone (Li et al., 2008). The IVD sustains compressive forces because of its elastic stiffness as well as the fluid pressures resulting from poroelasticity and the osmotic (swelling) effects (Stokes et al., 2011). It contains a large volume of extracellular matrix and a sparse population of cells distributed in the matrix. The extracellular matrix of IVD is composed largely of water (60-99\% by weight) and negatively charged proteoglycans, which confer a net negative charge on the tissue (Chen et al., 2002). As variations in disc loading lead to significant changes in disc hydration, changes in osmotic pressure play an important role in maintaining the physicochemical environment of IVD (Urban, 2002). Both hyper- and hypo-osmotic conditions are known to induce cell volume changes and calcium signaling in IVD cells (Pritchard et al., 2002; Pritchard and Guilak, 2004). A multitude of intracellular signaling events, such as alterations in intracellular ion composition and cell membrane potential, various second messenger cascades, phosphorylation of diverse target proteins, and altered gene expression, trigger the various cell volume regulatory mechanisms (Lang et al., 1998).

IVD cells can secrete a complex extracellular matrix that contains the highly sulfated proteoglycan aggrecan through its interaction with cations, and give the tissue its unique osmotic properties (Cheng et al., 2009). Previous studies have shown that fibrochondrocytes of the disc alter post-translational biosynthesis of proteoglycans, following exposure to hyper- or hypo-osmotic medium in both explant and isolated cell culture (Ishihara et al., 1997). A wide range of genes may be transcriptionally activated in multiple cell types following exposure to hypo- or hyper-osmotic conditions (Lang et al., 1998; Waldegger and Lang, 1998). Especially the genes encoding proteins related to cytoskeletal organization, ion transport, cytokines, and growth factors have been demonstrated to be regulated by osmotic conditions in cells of the IVD (Boyd et al., 2005). However, the molecular mechanisms regulating the response of IVD to osmotic pressure are still not well understood.

In the present study, we aimed to explore the molecular mechanism of the different responses of IVD cells following culture under hyper- and hypo-osmotic conditions, and to determine the target small molecules that exhibit similar effects with hyper- and hypo-osmotic response of IVD cells. The gene expression profile of human IVD cells following exposure to hyper- and hypo-osmolarity was downloaded from GEO.

\section{MATERIAL AND METHODS}

\section{Affymetrix microarray data}

The transcription profile of GSE1648 (Boyd et al., 2005) was obtained from a public functional genomics data repository, the GEO database (http://www.ncbi.nlm.nih.gov/geo/), which is based on the Affymetrix Human Genome U133A Array. Eleven samples including 4 hyper-osmotic stimuli, 4 iso-osmotic stimuli and 3 hypo-osmotic stimuli were applied to identify differentially expressed genes. Human IVD tissue was obtained from patients (average age of 51 years) undergoing discectomy prior to surgery for lumbar interbody fusion $(\mathrm{N}=3)$ or lumbar disc herniation $(\mathrm{N}=1)$. The iso-osmotic solution consisted of a defined cell culture 
medium $(293 \mathrm{mOsm} / \mathrm{kg} \mathrm{H} \mathrm{O})$. The hypo-osmotic solution consisted of the same cell culture media diluted with de-ionized water to a final osmolarity of $250 \mathrm{mOsm} / \mathrm{kg} \mathrm{H} \mathrm{H}_{2} \mathrm{O}$. The hyperosmotic solution consisted of the same cell culture media supplemented with sucrose to a final osmolarity of $450 \mathrm{mOsm} / \mathrm{kg} \mathrm{H} \mathrm{H}_{2} \mathrm{O}$. The three groups were used to investigate the response of the intervertebral disc to osmotic stimuli.

\section{Small molecule data}

The Connectivity Map can be used to find connections between small molecules sharing a mechanism of action, chemicals and physiological processes, and diseases and drugs (Lamb et al., 2006). It is the first installment of a reference collection of gene-expression profiles from cultured human cells treated with bioactive small molecules, together with pattern-matching software to mine these data. It includes 7056 genome-wide expression profiles representing 6100 individual treatment instances with 1309 bioactive small molecules. We downloaded all the profile data for further analysis.

\section{Pathway data}

KEGG (Kyoto Encyclopedia of Genes and Genomes) is a collection of online databases dealing with genomes, enzymatic pathways, and biological chemicals (Kanehisa, 2002). The PATHWAY database records networks of molecular interactions in cells and their variants, specific to particular organisms (http://www.genome.jp/kegg/).

\section{Analysis of differentially expressed genes (DEGs)}

For the GSE1648 dataset, the classical $t$-test method was used to identify DEGs. We used the limma (Smyth, 2004) package in R (Team, 2011) to preprocess the data of profile GSE1648. The raw expression datasets from all conditions were processed into expression estimates using the robust multiship analysis method (Irizarry et al., 2003) with the default setting implemented in Bioconductor, and then constructed the linear model. A P value less than 0.05 was chosen as the cutoff criterion.

\section{Pathway-enrichment analysis}

DAVID (Database for Annotation, Visualization and Integrated Discovery) (Huang et al., 2009), a high-throughput and integrated data-mining environment, which analyzes gene lists derived from high-throughput genomic experiments, was used to identify overrepresented pathways using the genes in our result. We selected the pathways with a P value less than 0.1 and count higher than 2 .

\section{RESULTS}

\section{Microarray data analysis}

Publicly available microarray dataset GSE1648 was obtained from GEO. We used 
the classical $t$-test method to identify the DEGs. A total of 243 genes were identified as significantly upregulated (136 genes) or downregulated (107 genes) in the samples following exposure to hyper-osmotic conditions compared to iso-osmotic conditions. A total of 368 genes were identified as significantly upregulated ( 86 genes) or downregulated ( 282 genes) in the samples following exposure to hypo-osmotic conditions compared to iso-osmotic conditions. A total of 431 genes were identified as significantly upregulated (335 genes) or downregulated (96 genes) in the samples following exposure to hyper-osmotic conditions compared to hypo-osmotic conditions. There were only two overlapping genes (RAP2A and GNA13) that expressed differentially in all osmotic conditions, which suggested they may play an important role in the response to osmotic stimuli in the intervertebral disc (Figure 1).

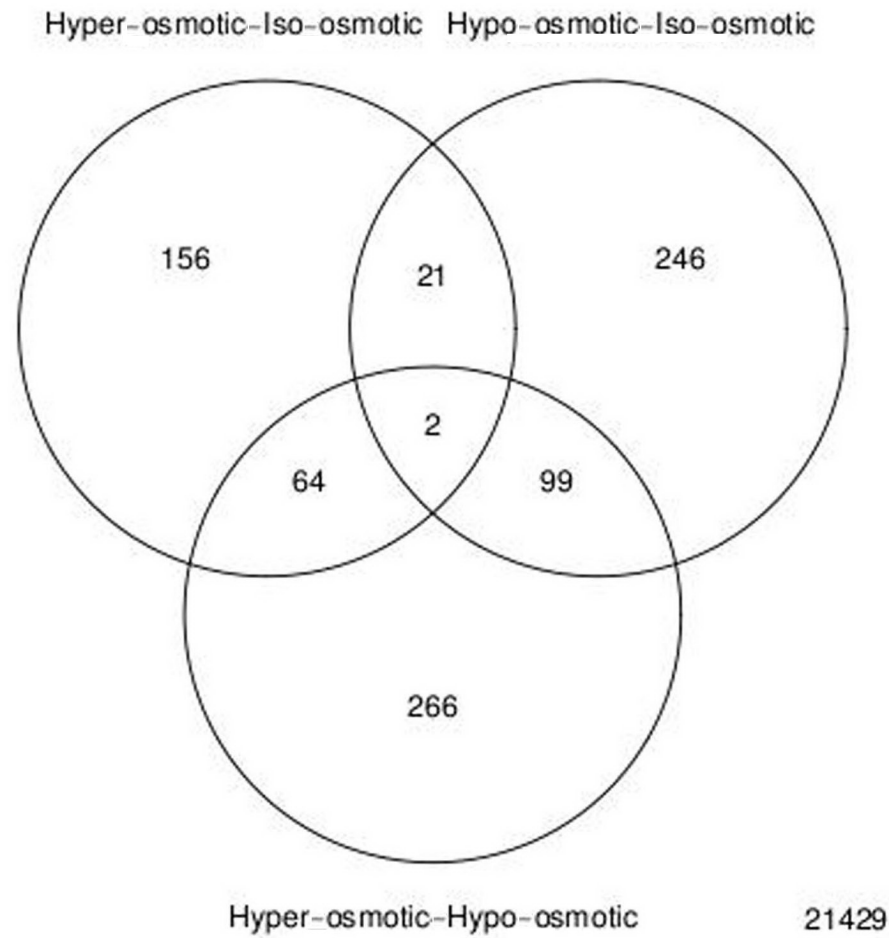

Figure 1. VENN graph displays the information of our data. Only two overlapping genes were identified in all three osmotic conditions.

\section{Pathway-enrichment analysis in different osmotic conditions}

To identify the relevant altered pathways in the response to osmotic stimuli in the intervertebral disc, we used the online biological classification tool DAVID to perform the pathwayenrichment analysis. To focus on the most significantly relevant pathways, a P value less than 0.05 and count higher than 2 were chosen as the thresholds. Five pathways were enriched in the three osmotic conditions (Table 1). The most significantly enriched pathway under hyper-osmotic conditions compared to iso-osmotic conditions was the spliceosome with $\mathrm{P}=0.033924$. The most signif- 
icantly enriched pathway under hypo-osmotic conditions compared to iso-osmotic conditions was drug metabolism with $\mathrm{P}=0.02044$. The most significantly enriched pathway under hyper-osmotic conditions compared to hypo-osmotic conditions was terpenoid backbone biosynthesis with $\mathrm{P}=$ 0.006589 . The other significant pathway was the TGF-beta signaling pathway with $\mathrm{P}=0.00795$.

Table 1. Enriched pathways.
\begin{tabular}{lllcc} 
& & & \\
\hline Class & Term & Description & Count & P \\
\hline Hyper-/iso-osmotic & hsa03040 & Spliceosome & 6 & 0.033924 \\
Hypo-/iso-osmotic & hsa00982 & Drug metabolism & 6 & 0.02044 \\
Hypo-/iso-osmotic & hsa03440 & Homologous recombination & 4 & 0.033569 \\
Hyper-/hypo-osmotic & hsa00900 & Terpenoid backbone biosynthesis & 4 & 0.006589 \\
Hyper-/hypo-osmotic & hsa04350 & TGF-beta signaling pathway & 8 & 0.00795 \\
\hline
\end{tabular}

\section{Identification of candidate small molecules}

To identify candidate small molecules able to cause similar effects with hyper- or hypo-osmotic conditions, we performed computational bioinformatics analysis of the derived gene signature using the Connectivity Map. A search against 6100 instances representing 1309 bioactive small molecules identified several compounds that may exhibit a similar effect to hyper- or hypo-osmotic response. The top 20 significant small molecules are listed in Table 2. We found that the small molecule thioridazine was significantly enriched in both groups with $\mathrm{P}$ value less than 0.001 .

Table 2. The top 20 significant small molecules in each group.

\begin{tabular}{llll}
\hline & \multicolumn{2}{c}{ Hyper- $v s$ iso-osmotic } \\
\hline Cmap name & \multicolumn{1}{c}{$\mathrm{P}$} & Cmap name & $\mathrm{P}$ \\
\hline Ouabain & 0 & Tanespimycin & 0.00036 \\
Digitoxigenin & 0 & Hexetidine & 0.00052 \\
Helveticoside & 0 & Thioridazine & 0.0006 \\
Digoxin & 0 & Metampicillin & 0.00134 \\
Lanatoside C & 0 & Betulinic acid & 0.00141 \\
Vorinostat & 0 & Wortmannin & 0.00149 \\
Thioridazine & 0 & NS-398 & 0.00154 \\
15-Delta prostaglandin J2 & 0 & Iohexol & 0.00165 \\
Trichostatin A & 0 & Morantel & 0.00234 \\
LY-294002 & 0 & Amantadine & 0.0028 \\
Loperamide & 0.00002 & Imidurea & 0.00324 \\
Proscillaridin & 0.00004 & Myosmine & 0.00433 \\
Parthenolide & 0.00012 & Adiphenine & 0.00435 \\
Terfenadine & 0.0002 & Meropenem & 0.00525 \\
Withaferin A & 0.0004 & Propantheline bromide & 0.00549 \\
Prochlorperazine & 0.00044 & Clebopride & 0.00559 \\
Suloctidil & 0.0007 & Alpha-estradiol & 0.00598 \\
Digoxigenin & 0.00104 & Nordihydroguaiaretic acid & 0.00663 \\
MG-262 & 0.00136 & Perhexiline & 0.00716 \\
7-Aminocephalosporanic acid & 0.00163 & Coralyne & 0.00718 \\
\hline
\end{tabular}

\section{DISCUSSION}

The IVD is a heterogeneous structure that serves as the spine's shock absorbing system, 
which protects the vertebrae, brain, and other structures. IVD cells experience a broad range of physical stimuli under physiologic conditions, including alterations in their osmotic environment (Boyd et al., 2005). In this study, we investigated the molecular mechanism of IVD cells in the response to osmotic pressure, and to find therapeutic small molecules that have similar effect with hyper-osmotic and hypo-osmotic response. The results of this study suggest that RAP2A and GNA13 may play an important role in the response to osmotic stimuli in the intervertebral disc. In addition, we found that thioridazine was significantly enriched in our result, which suggested that it may be a potential drug to protect IVD cells from changes of osmotic pressure.

RAP2A belongs to the RAS subfamily of small GTP-binding proteins, which are considered to control cell growth, differentiation and survival (Bokoch, 1993; Burgering and Bos, 1995). To date, there is no direct evidence of RAP2A ever regulating osmotic pressure; however, previous studies have demonstrated that Rap1 plays a direct role in the activation of guanylyl cyclase during the response to hyper-osmotic conditions (Kang et al., 2002). Because Rap2 and Rap1 proteins are 60\% identical to each other (Reuther and Der, 2000; Takai et al., 2001), perhaps they share some common functions. Therefore, we hypothesize that Rap2 may play a role in the response to osmotic conditions too. Certainly, further studies are needed to verify this notion.

GNA13, a member of guanine nucleotide binding proteins (G proteins), are involved in transmitting chemical signals outside the cell, and causing changes inside the cell. They communicate signals from many hormones, neurotransmitters, and other signaling factors. Osmotic pressure is known to induce cell volume changes and calcium signaling in IVD cells (Pritchard et al., 2002; Pritchard and Guilak, 2004). Davis et al. (1992) suggest that a G protein plays a key role in the transduction of the shrinkage signal to the $\mathrm{Na}-\mathrm{H}$ exchanger via a pathway. Besides, inhibitors of $\mathrm{G}$ proteins have been shown to blunt swelling-induced osmolyte efflux (Ruhfus et al., 1996), as well as increase intracellular $\mathrm{Ca}^{2+}$ concentration (Arora et al., 1994) and mitogen-activated protein kinase (MAPK) activity (Noe et al., 1996). These results suggest that $\mathrm{G}$ proteins do regulate some effects of cell volume. Our results suggest that GNA13 expressed differentially in the response to hyper- and hypo-osmotic conditions, which is in line with previous studies.

From the result of pathway-enrichment analysis in different osmotic conditions, we found that most of the enriched pathways are related to protein biosynthesis and signal transduction. These results indicate that physiological metabolism changed under different osmotic conditions.

The identification of a group of small molecules with potential therapeutic efficacy for regulating the osmotic pressure of IVD cells is an important implication of our study. Through computational bioinformatics analysis using the Connectivity Map, we identified hundreds of small molecules capable of causing similar effects as hyper- or hypo-osmotic conditions with a $\mathrm{P}$ value less than 0.05 . In particular, we found that the small molecule thioridazine was significantly enriched in both osmotic conditions with a $P$ value less than 0.001 . We are not aware of any reports of the evaluation or use of this compound in systemic therapies for osmotic pressure change. Although this compound has been widely used in the treatment of schizophrenia and psychosis, there is a lack of knowledge as to its effects on IVD. Because of previous concerns regarding the potential risk of cardiotoxicity and retinopathy at high doses, this drug is not regularly prescribed and reserved for some patients. Given the previously widespread use of this drug for the treatment of mental disease, it is unlikely that they will be exploited as therapeutics by themselves, but may have promise for use in combination therapy.

Our results warrant further study and should generate hypotheses for laboratory-, patient- 
or population-based studies. Laboratory studies are needed to explore and identify the potential mechanisms by which this small molecule exerts its effects in regulating the osmotic pressure of IVD cells. Also, clinical trials to evaluate the effect of concomitant drugs on responses to treatment of osmotic pressure changes of IVD cells should be considered. Finally, epidemiological studies to examine the effects of concomitant therapy on the outcomes of IVD treatment are also justified.

\section{CONCLUSIONS}

In conclusion, we explored the molecular mechanism of the different responses of IVD cells following culture under hyper- and hypo-osmotic conditions. Hundreds of genes were identified as significantly upregulated or downregulated in the samples following exposure to hyper- and hypo-osmotic conditions. RAP2A and GNA13 were found to be expressed differentially in all three osmotic conditions. In addition, we used computational bioinformatics to identify potential therapeutic drug candidates capable of regulating osmotic pressure changes in IVD cells. However, further experiments are still needed to confirm our conclusion.

\section{REFERENCES}

Arora PD, Bibby KJ and McCulloch CA (1994). Slow oscillations of free intracellular calcium ion concentration in human fibroblasts responding to mechanical stretch. J. Cell Physiol. 161: 187-200.

Bokoch GM (1993). Biology of the Rap proteins, members of the ras superfamily of GTP-binding proteins. Biochem. J. 289: 17-24.

Boyd LM, Richardson WJ, Chen J, Kraus VB, et al. (2005). Osmolarity regulates gene expression in intervertebral disc cells determined by gene array and real-time quantitative RT-PCR. Ann. Biomed. Eng. 33: 1071-1077.

Burgering BM and Bos JL (1995). Regulation of Ras-mediated signalling: more than one way to skin a cat. Trends Biochem. Sci. 20: 18-22.

Chen J, Baer AE, Paik PY, Yan W, et al. (2002). Matrix protein gene expression in intervertebral disc cells subjected to altered osmolarity. Biochem. Biophys. Res. Commun. 293: 932-938.

Cheng CC, Uchiyama Y, Hiyama A, Gajghate S, et al. (2009). PI3K/AKT regulates aggrecan gene expression by modulating Sox9 expression and activity in nucleus pulposus cells of the intervertebral disc. J. Cell Physiol. 221: 668-676.

Davis BA, Hogan EM and Boron WF (1992). Role of G proteins in stimulation of Na-H exchange by cell shrinkage. Am. J. Physiol. 262: C533-C536.

Huang W, Sherman BT and Lempicki RA (2009). Systematic and integrative analysis of large gene lists using DAVID bioinformatics resources. Nat. Protoc. 4: 44-57.

Irizarry RA, Hobbs B, Collin F, Beazer-Barclay YD, et al. (2003). Exploration, normalization, and summaries of high density oligonucleotide array probe level data. Biostatistics 4: 249-264.

Ishihara H, Warensjo K, Roberts S and Urban JP (1997). Proteoglycan synthesis in the intervertebral disk nucleus: the role of extracellular osmolality. Am. J. Physiol. 272: C1499-C1506.

Kanehisa M (2002). The KEGG database. Novartis Found. Symp. 247: 91-101.

Kang R, Kae H, Ip H, Spiegelman GB, et al. (2002). Evidence for a role for the Dictyostelium Rap1 in cell viability and the response to osmotic stress. J. Cell Sci. 115: 3675-3682.

Lamb J, Crawford ED, Peck D, Modell JW, et al. (2006). The Connectivity Map: using gene-expression signatures to connect small molecules, genes, and disease. Science 313: 1929-1935.

Lang F, Busch GL, Ritter M, Volkl H, et al. (1998). Functional significance of cell volume regulatory mechanisms. Physiol. Rev. 78: 247-306.

Li S, Duance VC and Blain EJ (2008). Zonal variations in cytoskeletal element organization, mRNA and protein expression in the intervertebral disc. J. Anat. 213: 725-732.

Noe B, Schliess F, Wettstein M, Heinrich S, et al. (1996). Regulation of taurocholate excretion by a hypo-osmolarityactivated signal transduction pathway in rat liver. Gastroenterology 110: 858-865.

Pritchard S and Guilak F (2004). The role of F-actin in hypo-osmotically induced cell volume change and calcium signaling in anulus fibrosus cells. Ann. Biomed. Eng. 32: 103-111. 
Pritchard S, Erickson GR and Guilak F (2002). Hyperosmotically induced volume change and calcium signaling in intervertebral disk cells: the role of the actin cytoskeleton. Biophys. J. 83: 2502-2510.

Reuther GW and Der CJ (2000). The Ras branch of small GTPases: Ras family members don't fall far from the tree. Curr. Opin. Cell Biol. 12: 157-165.

Ruhfus B, Tinel H and Kinne RK (1996). Role of G-proteins in the regulation of organic osmolyte efflux from isolated rat renal inner medullary collecting duct cells. Pflugers Arch. 433: 35-41.

Smyth GK (2004). Linear models and empirical Bayes methods for assessing differential expression in microarray experiments. Stat. Appl. Genet. Mol. Biol. 3: Article3.

Stokes IA, Laible JP, Gardner-Morse MG, Costi JJ, et al. (2011). Refinement of elastic, poroelastic, and osmotic tissue properties of intervertebral disks to analyze behavior in compression. Ann. Biomed. Eng. 39: 122-131.

Takai Y, Sasaki T and Matozaki T (2001). Small GTP-binding proteins. Physiol. Rev. 81: 153-208.

Team RDC (2011). R: A Language and Environment for Statistical Computing. R Foundation for Statistical Computing, Vienna.

Urban JP (2002). The role of the physicochemical environment in determining disc cell behaviour. Biochem. Soc. Trans. 30: $858-864$.

Waldegger S and Lang F (1998). Cell volume and gene expression. J. Membr. Biol. 162: 95-100. 